IRRITABLE BOWEL DISEASE

\title{
Eosinophil granulocytes are activated during the remission phase of ulcerative colitis
}

\author{
M Lampinen, A Rönnblom, K Amin, G Kristjansson, F Rorsman, P Sangfelt, B Säfsten, \\ M Wagner, A Wanders, O Winqvist, M Carlson
}

See end of article for authors' affiliations

Correspondence to: Dr M Lampinen, Department of Medical Sciences, Clinical Chemistry and Medicine, University Hospital, S-75 85 Uppsala, Sweden; maria.lampinen@ medsci.uu.se

Revised version received 28 April 2005 Accepted for publication 7 May 2005 Published online first 10 May 2005 ....................
Aim: The aim of this study was to establish a method of investigating intestinal eosinophil and neutrophil granulocytes by flow cytometry, and to compare the distribution and activity of these cells in different stages of ulcerative colitis (UC).

Methods: Biopsy samples were taken from six locations of the entire colon and from the terminal ileum in 10 patients with active total UC, 10 patients with inactive total UC, eight patients with active distal UC, and 11 control subjects. Cell suspensions from biopsies and from peripheral blood were incubated with fluorophore conjugated monoclonal antibodies. The use of scatter plot-gating and specific antibodies was established in a flow cytometry assay.

Results: Eosinophils were more numerous and more active in patients with active UC than in controls. Interestingly, during inactive UC, the number of activated eosinophils was even larger. Eosinophil activity was high in the rectum of patients with distal colitis but was also slightly elevated in the proximal colon. Neutrophils were increased in number and activity during active but not inactive UC. In patients with distal colitis, activated neutrophils were only found in the sigmoid colon and rectum.

Conclusion: With this method, we confirm that neutrophils participate in the inflammatory process during active UC, and that they express a resting phenotype during remission. The finding of activated eosinophils in inflamed intestine strengthens the view of these cells as proinflammatory and tissue damaging. Nevertheless, our new finding of high eosinophil activation during inactive UC suggests that eosinophils play a role in repair of injured epithelium.
U lcerative colitis (UC) is a chronic inflammatory disease of unknown aetiology. It is characterised by periods of exacerbation (active disease) and remission (inactive disease); the main symptoms during exacerbation are diarrhoea and rectal bleeding. The inflammation affects the colonic mucosa, with accumulation of lymphocytes, monocytes, mast cells, and neutrophil and eosinophil granulocytes. The rectum is invariably involved, and the disease may remain as a proctitis or extend proximally to the sigmoid or descending colon (distal colitis) or to the entire length of the colon (total colitis). ${ }^{1}$

Eosinophils are proinflammatory cells with the capacity to produce and release toxic proteins, such as eosinophil cationic protein (ECP), eosinophil protein X (EPX), and eosinophil peroxidase (EPO), as well as several proinflammatory cytokines such as interleukin (IL)- $1 \alpha^{2}{ }^{2}$ IL- $2,{ }^{3}$ IL- $5,{ }^{4}$ IL-3, IL-4, IL-8, tumour necrosis factor $\alpha$ and RANTES, ${ }^{5}$ reactive oxygen metabolites, and lipid mediators. Besides their ability to kill invading parasites, eosinophils take part in immunological events by releasing different mediators ${ }^{6}$; it has also been suggested that they may act as antigen presenting cells with an ability to stimulate $\mathrm{T}$ cell proliferation and activation. ${ }^{7}$ Eosinophils are implicated in the inflammatory process of bronchial asthma and allergic disorders $^{6}$ and have a critical role in the pathophysiology of eosinophil associated gastrointestinal disease. ${ }^{8}$

Recent studies indicate that eosinophils may also be involved in remodelling and tissue repair through fibroblast stimulation by release of ECP and transforming growth factor (TGF) $-\beta .^{9}{ }^{10}$

The role of eosinophil granulocytes in UC remains obscure. These cells are normally present in the intestinal mucosa, participating in host defence. ${ }^{8}$ However, the number of eosinophils is highly increased in patients with $\mathrm{UC}^{11}$ and increased levels of eosinophil granule proteins have been detected in intestinal perfusion fluid ${ }^{12}$ and in faeces ${ }^{13}$ from patients with UC. Elevated concentrations of eosinophil chemotactic factors ${ }^{14}$ and increased expression of adhesion molecules $^{15}$ in the colon during active UC indicate that eosinophils are actively recruited to the inflamed mucosa.

The involvement of neutrophil granulocytes in the pathogenesis of UC is more established; in fact, infiltration of the colonic mucosa by neutrophils is regarded as a hallmark for activity in this disease. ${ }^{16}{ }^{17}$ Neutrophils are recruited from the circulation to take part in the defence against infectious agents but they may also cause tissue destruction in the host by secretion of toxic granule proteins and reactive oxygen species. ${ }^{6}$ Release of neutrophil granule proteins has been observed in both the mucosa and lumen of the intestine in patients with active UC and proctitis. ${ }^{18} 19$

Previous studies at our laboratory have demonstrated the presence and activity of eosinophils by measurement of eosinophil products in intestinal perfusion fluid, faeces, and blood. ${ }^{12}{ }^{13}$ We believe that these assays provide a good reflection of eosinophil activity in intestinal disease. However, in the present study, we aimed to look more closely at the cell itself and investigate eosinophils at the site of inflammation. For this purpose, we have established a sensitive and stable flow cytometric assay and we have used this assay to study granulocytes from intestinal biopsy

\footnotetext{
Abbreviations: UC, ulcerative colitis; $\mathrm{mAb}$, monoclonal antibody; $\mathrm{ECP}$, eosinophil cationic protein; EPX, eosinophil protein X; EPO, eosinophil peroxidase; TGF, transforming growth factor; FACS, fluorescence activated cell sorting; FITC, fluorescein isothiocyanate; PE, phycoerythrin; PerCP, peridinin chlorophyll protein; MPO, myeloperoxidase; MFI, mean fluorescence intensity; IL, interleukin
} 
samples and peripheral blood. Using this method we can evaluate the number of activated versus resting eosinophils as well as the degree of activation of individual cells, thus obtaining a better understanding of the role of eosinophils during different stages of UC. In addition, immunohistochemical staining was performed on intestinal biopsy samples.

The overall aim of this study was to quantify and assess the activity of eosinophil granulocytes in different stages of the disease process in patients with UC and in healthy individuals, and also to compare inflamed and non-inflamed parts of the colon of patients with distal colitis. The activity of neutrophil granulocytes was studied in the same patients and was considered in relation to the findings regarding eosinophils.

\section{PATIENTS AND METHODS}

\section{Patients and control group}

Clinical and demographical characteristics of patients and controls are presented in table 1 .

The diagnoses were based on established clinical, endoscopic, and histological criteria. ${ }^{20}$ Patients were considered to be in a phase of inactive disease if they had no clinical symptoms of disease activity and the endoscopic picture was normal or at most showed a slight disturbance of mucosal vessels. Patients with clinical symptoms (at least 2-4 soft stools/day and blood in the faeces) and endoscopic signs of inflammation with a score of 3-4 on the Binder scale ${ }^{21}$ (granularity, friability, pus or blood, ulcers) were considered to have active disease. Controls were recruited among patients examined for anaemia $(n=5)$ or were healthy volunteers $(n=6)$. Biopsy samples were taken during colonoscopy (Olympus 160 AL endoscope with standard endoscopy forceps) after bowel preparation, as for routine flexible rectosigmoid endoscopy: two days of diet restriction and an oral purgative in the morning and afternoon on the day before the examination. The project was approved by the ethics committee of the Medical Faculty, Uppsala University, and all patients gave their informed consent to participation in the study.

\section{Collection and preparation of samples}

During colonoscopy, four adjacent biopsy samples were taken from each of seven different locations in all patients and control subjects: the terminal ileum, caecum, right and left flexures of the colon, descending colon, sigmoid colon, and rectum. Two of the samples from each location were sent for routine histological analysis. The remaining two samples were immediately transferred into tubes filled with physiological saline solution at room temperature, and were further processed within one hour. Peripheral blood was also collected from all participating patients and control subjects.
Biopsy samples from each location were handled separately for comparison of the different parts of the colon. Single cell suspensions of biopsy cells were obtained using a loosely fit glass homogeniser, and cells were then washed twice with a buffer assigned for fluorescence activated cell sorting (FACS) containing $0.05 \% \mathrm{NaN}_{3}, 0.1 \%$ bovine serum albumin, and $0.4 \%$ trisodium citrate dihydrate in phosphate buffered saline. Heparinised peripheral blood from the same individuals was haemolysed with a $0.83 \%$ ammonium chloride solution and washed twice in the FACS buffer to obtain a suspension of blood leucocytes. Both types of cell suspensions were incubated with fluorophore conjugated monoclonal antibodies (mAbs) for 30 minutes at room temperature in the dark. After a final wash, cells were suspended in $500 \mu \mathrm{l}$ of the FACS buffer and analysed.

\section{Antibodies}

Mouse-antihuman mAbs conjugated to fluorescein isothiocyanate (FITC), phycoerythrin (PE), or peridinin chlorophyll protein (PerCP) were used for all antigens. Isotype matched control labelling was also performed using fluorophore conjugated mouse antihuman IgM $\mathrm{I}$ and $\operatorname{IgG} 2 \mathrm{~b} \kappa$ as controls for non-specific staining. All antibodies used for flow cytometry were purchased from Becton Dickinson (BD) Biosciences/Pharmingen (San Diego, California, USA). AntiCD16 conjugated micro beads were purchased from Miltenyi Biotech, GmbH (Germany).

\section{Flow cytometry assay}

The flow cytometry assay was performed on a two laser FACS Calibur Cytometer (BD Immunocytometry Systems, San José, California, USA). Three types of fluorophores were used to enable analysis of three different antigens in the same sample: FITC, emitting $519 \mathrm{~nm}$ green colour in FLl; PE, emitting $578 \mathrm{~nm}$ yellow-green colour in FL2; and PerCP, emitting $675 \mathrm{~nm}$ red colour in FL3. Fluorescence measurements were collected using a logarithmic amplifier; forward and side scatter was studied using a linear amplifier. Ten thousand cells were counted and analysed in each sample. For data analyses, Cell Quest Pro software (Becton Dickinson) was used.

\section{Identification of cells and markers of activation}

Eosinophil and neutrophil granulocytes from peripheral blood or biopsy samples were gated by their forward and side scatter properties and further identified by surface markers (fig 1). CD9 has previously been used as a marker for eosinophils ${ }^{22}$ but in our setting the specificity needed to be further increased. We therefore used CD9 combined with anti-CDw125, which is expressed on eosinophils and basophils. ${ }^{23}$ The specificity of these markers was confirmed by analysis on an EPICS XL flow cytometer (Coulter

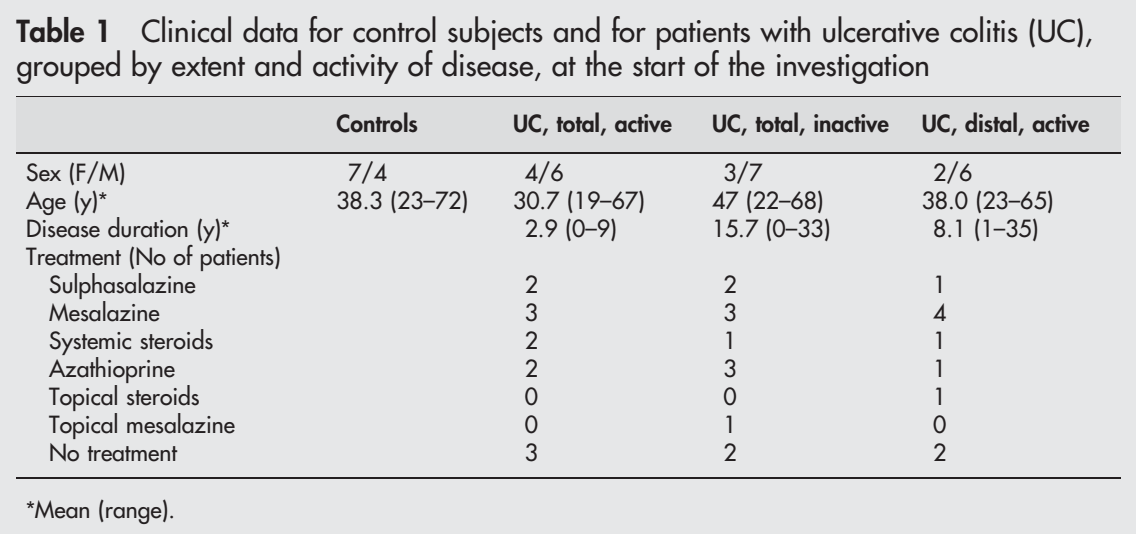


A

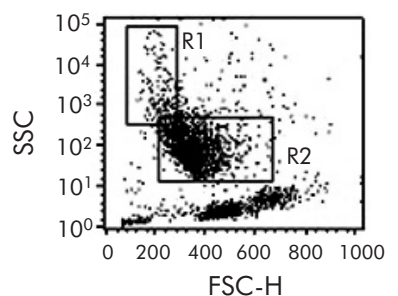

C

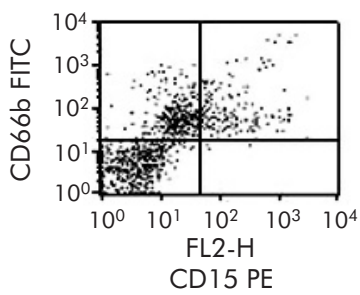

$\mathrm{E}$

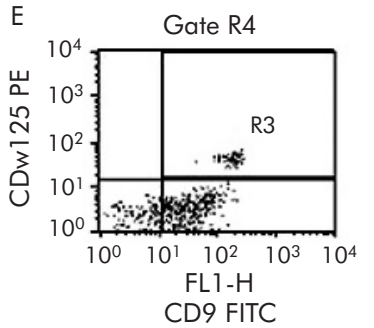

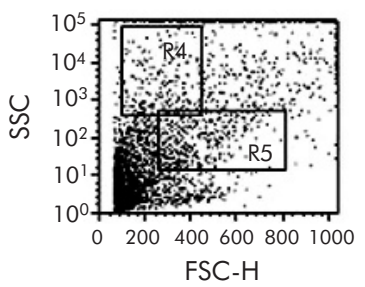



F Gate R4 and R3



Figure 1 Representative plots describing the procedure of cell identification by flow cytometry. Cell populations are selected for further analysis by drawing gates (numbered R1, R2, etc) around the cells of interest in the forward/side scatter plot. Gated cells are then transferred to new plots for evaluation of their expression of specific markers. (A) Peripheral blood leucocytes: gating of eosinophil (R1) and neutrophil (R2) granulocytes. (B) Intestinal biopsy: gating of eosinophil (R4) and neutrophil (R5) granulocytes. (C) Intestinal neutrophils gated by R5. The upper right quadrant contains CD66b positive neutrophils. (D) Intestinal neutrophils gated by R5. Isotype matched control monoclonal antibodies (mAbs) for CD66b and CD15 (mouse antihuman IgMk fluorescein isothiocyanate (FITC) and phycoerythrin (PE), respectively). (E) Intestinal eosinophils gated by R4. Cells in the upper right quadrant expressing CD9 and CDw1 25 are gated (R3) for evaluation of CD44 (F). (F) Expression of CD44 on eosinophils gated by R4 and R3 compared with isotype matched control $\mathrm{mAb}$ (mouse anti-human IgG2b\% peridinin chlorophyll protein (PerCP)). A gate (MI) is set on the eosinophil population with an expression of CD44 exceeding isotype control staining.

Electronics, Hialeah, Florida, USA) with optical filters for identification of eosinophils. To ensure that the markers were expressed on all eosinophils, we purified peripheral blood eosinophils by Percoll centrifugation and subsequent magnetic bead sorting (removal of neutrophils with anti-CD16). The purified eosinophils were analysed on the FACS Calibur. We found that $100 \%$ of the eosinophils expressed both CD9 and CDw125, and therefore concluded that these markers are suitable for identification of eosinophils. CD44, the receptor for hyaluronic acid, was used as a marker of eosinophil activation. ${ }^{24}$ Another indicator of eosinophil activity is mean fluorescence intensity (MFI) of CD9, which is high on resting cells and decreases on activation, probably because of shedding. ${ }^{25}$ CD15 is expressed on both eosinophils and neutrophils but the MFI of this molecule is 10-100 times higher on neutrophils, ${ }^{26}$ and the neutrophil population is therefore easily distinguished from eosinophils. CD66b is stored in the secondary granules of neutrophils and mobilised to the surface on activation. Accordingly, the MFI

of this molecule was used as a measure of neutrophil activation. ${ }^{27} 28$

\section{Immunohistochemistry}

Immunohistochemical analyses were performed on biopsy specimens from six patients with active total UC, five patients with inactive total UC, and five patients with active distal colitis. Neutrophil granulocytes were identified by a polyclonal $\mathrm{Ab}$ to myeloperoxidase (MPO) (obtained from the Department of Medical Sciences, Clinical Chemistry, University of Uppsala, Sweden), and the activation marker CD66b (Research Diagnostics Inc, Flanders, New Jersey, USA) was subsequently stained on the same sections to ensure that CD66b was expressed on neutrophils. Monoclonal antibodies to EPO (Department of Medical Sciences, Clinical Chemistry, University of Uppsala, Sweden) and CD9 (Dakocytomation, Glostrup, Denmark) were used to identify eosinophil granulocytes. Sections cut from wax embedded blocks (prepared for routine histological analysis) were deparaffinised in xylene, rehydrated through decreasing concentrations of alcohol, and rinsed in Tris buffered saline $(\mathrm{pH}$ 7.6). Sections were incubated with mAbs overnight in a humid chamber at room temperature and processed as previously described. ${ }^{29}$ The antigen-antibody complex was visualised using a commercial APAAP kit (K621; Dako, Glostrup, Denmark) and a fast red substrate, according to the instructions given in the manual. Samples were then counterstained with Mayer's haematoxylin (Merck D-6071, Darmstadt, Germany). Sections were examined with a Leica DRMB microscope.

\section{Statistical evaluation}

Kruskal-Wallis ANOVA and the Mann-Whitney U test were used to evaluate statistical differences between the groups of patients. For paired analyses, we used Friedman ANOVA and the Wilcoxon matched pairs test. A p value $<0.05$ was adopted as significant. All calculations were performed on a personal computer using the statistical software Statistica (Statsoft Inc, Tulsa, Oklahoma, USA).

\section{RESULTS}

\section{Method evaluation}

We tested and evaluated different cell markers and markers of activation, and optimised the procedure of cell gating. The resulting method has been used throughout the study, and is described in the methods section.

In four of the first experiments, we evaluated the reproducibility of the method by comparing several biopsy samples from the same area of the colon. We analysed the distribution and activation of neutrophils, eosinophils, CD $4^{+}$, and $\mathrm{CD}^{+} \mathrm{T}$ lymphocytes, B lymphocytes, macrophages, and enterocytes by flow cytometry, and found only negligible differences between adjacent samples (not shown).

\section{Intestinal eosinophil granulocytes}

Immunohistochemical staining for EPO and CD9 revealed large numbers of eosinophils in the lamina propria of patients with active total UC (fig 2A, B), and high release activity of EPO was detected in these patients. The number of eosinophils was only slightly decreased in biopsy specimens from patients with inactive total UC but release of EPO into the tissue was less pronounced in these patients (fig 2C, D).

\section{Markers of eosinophil activation}

There were no significant differences in the MFI of CD44 on eosinophils from control subjects, patients with active total UC, and patients with inactive total UC (not shown). The percentage of activated eosinophils however was higher in patients with active total UC than in control subjects. 

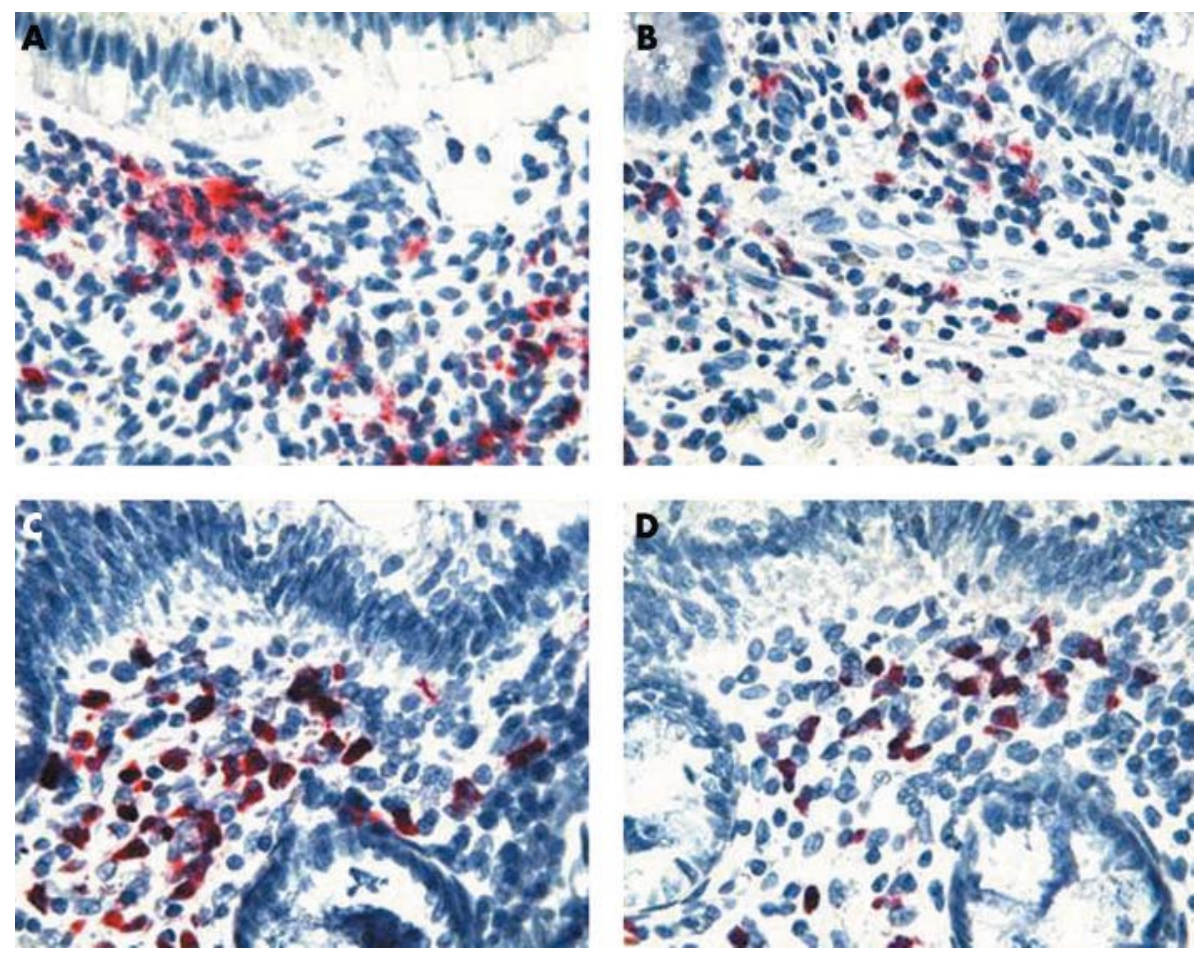

Figure 2 Immunohistochemical identification of eosinophils in colonic biopsy specimens from patients with active total ulcerative colitis (UC) (A, B) and inactive total UC (C, D). (A, C) Staining with the eosinophil marker eosinophil peroxidase; (B, D) staining with CD9. Included are two patients, representative of six patients with active total UC and five patients with inactive total UC, respectively. Cells were stained with APAAP and fast red substrate and counterstained with Mayer's haematoxylin. Original magnification $\times 340$.

Interestingly, this proportion was further increased in patients with inactive UC, even in the terminal ileum (fig 3 ).

We found a tendency towards a lower MFI of CD9 on eosinophils from patients with active total UC than on those from control subjects, and significantly lower expression of CD9 on eosinophils from patients with inactive total UC compared with control subjects. Expression of CD9 was low in the terminal ileum in all three groups (fig 4).

Patients with active distal colitis had a significantly larger percentage of activated eosinophils in the rectum than in the

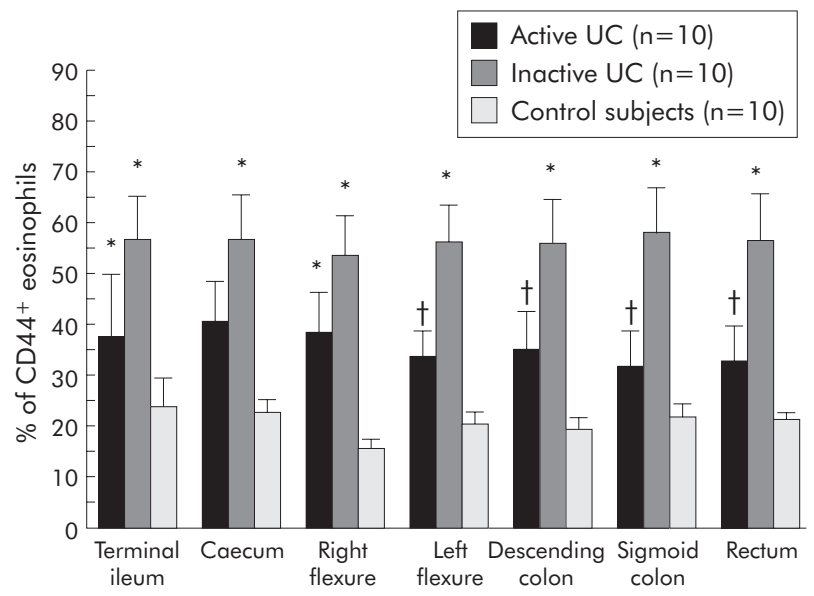

Figure 3 Percentage numbers of activated $\left(C D 44^{+}\right)$eosinophils. Values significantly different from those of control subjects are indicated above the respective columns ( ${ }^{*} p<0.05$ ). Significant differences between patients with active total ulcerative colitis (UC) and inactive total UC are indicated $(t p<0.05)$. Results are expressed as mean (SEM). KruskalWallis ANOVA and the Mann-Whitney $U$ Test were used for statistical evaluation. proximal parts of the colon, and a tendency towards a higher percentage in the sigmoid colon (fig 5). Compared with control subjects, the proportion of activated eosinophils was significantly higher $(\mathrm{p}<0.05)$ not only in the distal colon but also in the right flexure and terminal ileum in patients with distal colitis.

\section{Peripheral blood eosinophils}

There was no significant difference in MFI of CD44 on peripheral blood eosinophils between control subjects and patients, either with active or inactive disease (not shown).

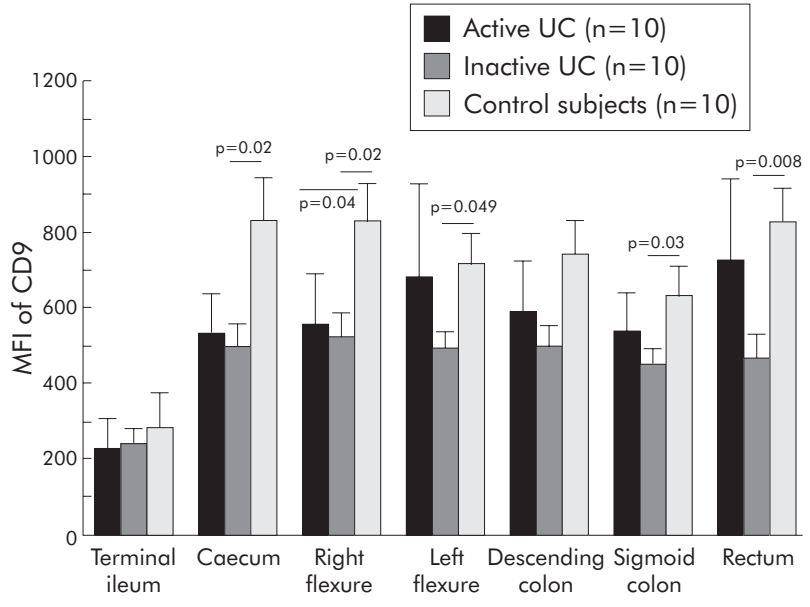

Figure 4 Mean fluorescence intensity (MFI) of CD9 on eosinophils in active total ulcerative colitis (UC), inactive total UC, and in controls. Significant differences between patient groups are indicated. Results are expressed as mean (SEM). Kruskal-Wallis ANOVA and the MannWhitney $U$ test were used for statistical evaluation. 


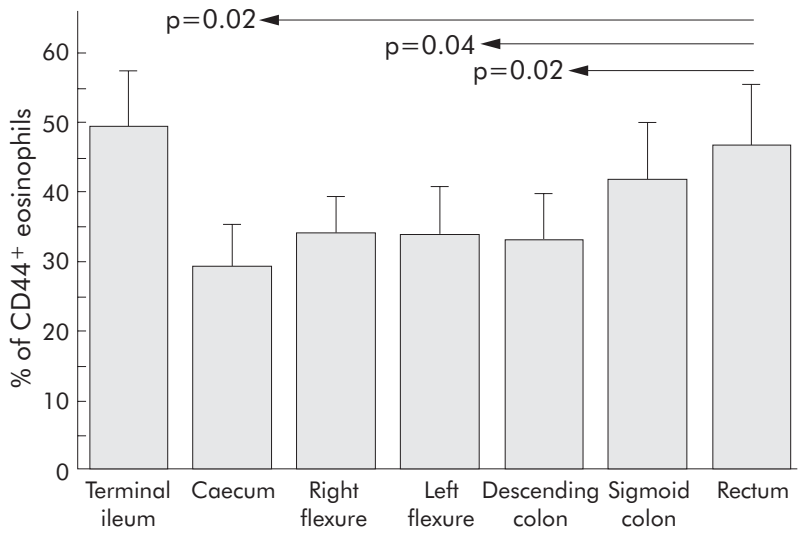

Figure 5 Percentage numbers of activated $\left(C D 44^{+}\right)$eosinophils from patients with active distal ulcerative colitis $(U C)(n=8)$. Significant differences (Friedman ANOVA and the Wilcoxon matched pairs test) between different intestinal locations are indicated. Results are expressed as mean (SEM).

The percentage of $\mathrm{CD}_{4} 4^{+}$eosinophils was significantly $(p<0.05)$ higher on peripheral blood eosinophils from patients with inactive UC than from patients with active UC and control subjects (80.1 (SEM 6.9)\%, 53.4 (12.5)\%, and $57.4(8.1) \%$, respectively). The MFI of CD9 was significantly $(p<0.05)$ higher on peripheral blood eosinophils from control subjects than on those from patients with active and inactive UC (525 (SEM 118), 203 (42.1), 248 (22.3), respectively).

\section{Intestinal neutrophil granulocytes}

Immunohistochemistry showed intensive staining with MPO (fig 6A) and CD66b (fig 6B) in the lamina propria of patients with active total UC, indicating large numbers of activated neutrophils in the tissue. In biopsy specimens from patients

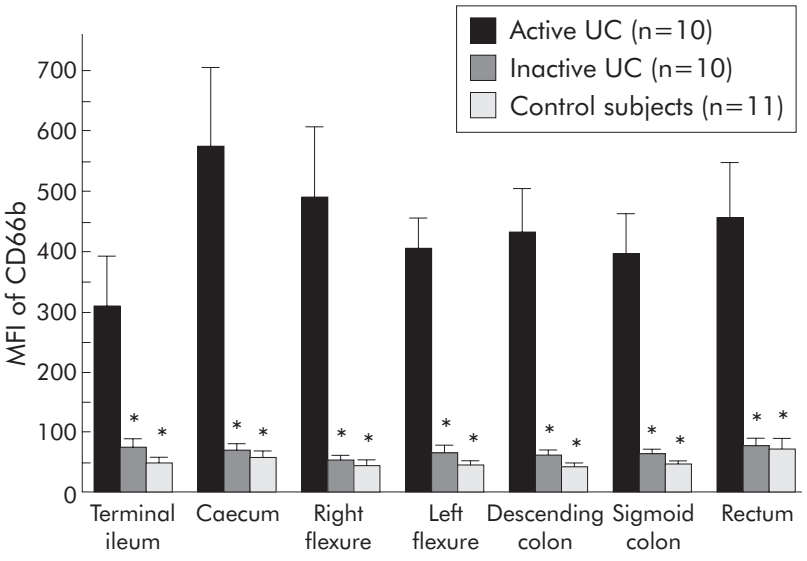

Figure 7 Mean fluorescence intensity (MFI) of CD66b on intestinal neutrophils (mean (SEM)). Values significantly different from those in active total ulcerative colitis (UC) are indicated above the respective columns ( $\left.{ }^{*} p<0.05\right)$. Kruskal-Wallis ANOVA and the Mann-Whitney U test were used for statistical evaluation.

with inactive total UC, there were only a few MPO positive cells (fig 6C), and CD66b expression was low (fig 6D).

\section{Expression of CD66b}

The activity of neutrophil granulocytes was assessed as expression of CD66b by flow cytometry. The MFI of CD66b was significantly higher on neutrophils from patients with active total UC than from those with inactive total UC and control subjects (fig 7). There were no significant differences in CD66b expression between the different intestinal locations either in patients with total UC or control subjects.

There was high expression of CD66b on neutrophils from the sigmoid colon and rectum in patients with active distal colitis whereas expression of CD66b in the proximal parts of
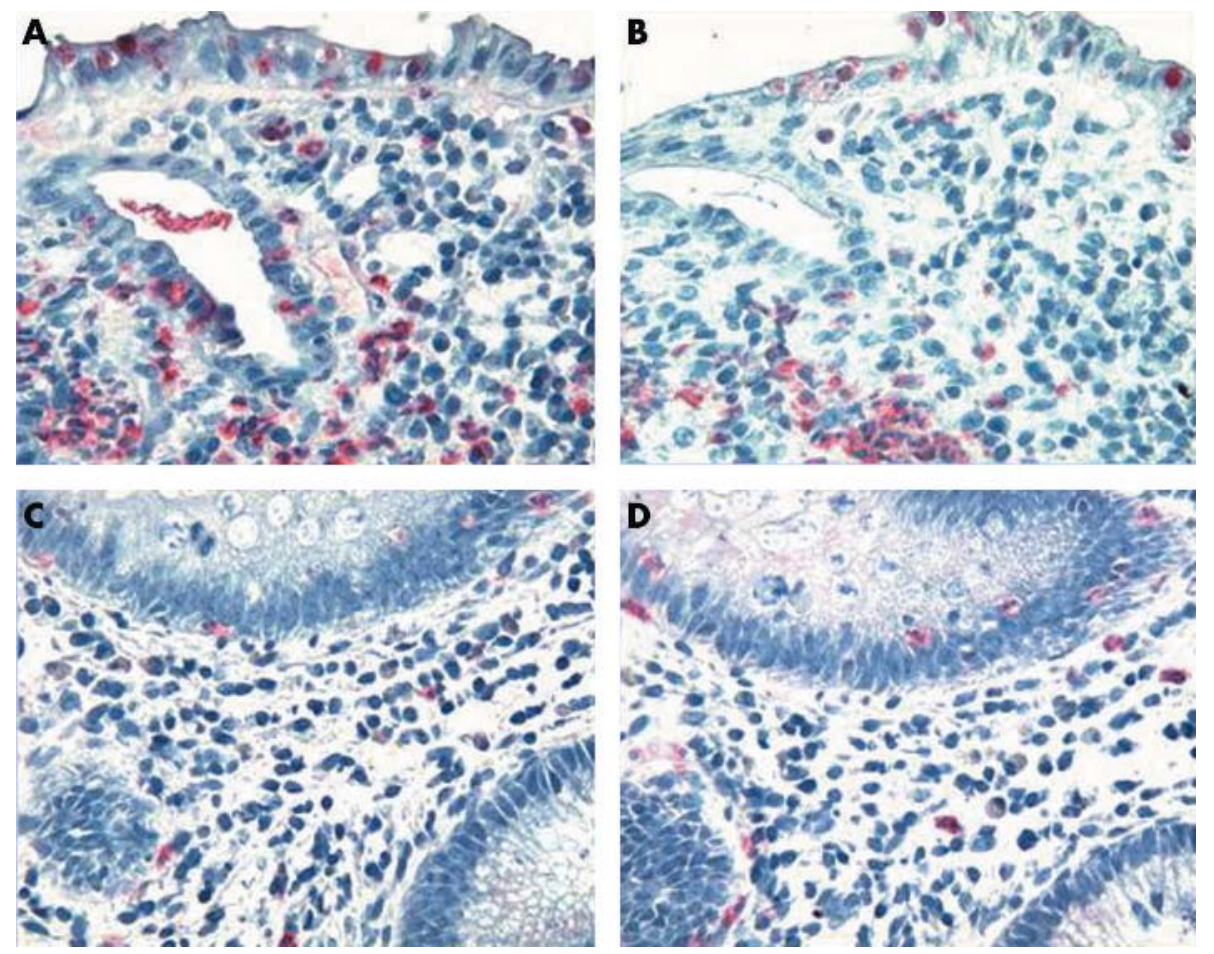

Figure 6 Immunohistochemical identification of neutrophils in colonic biopsy specimens from patients with active total ulcerative colitis (UC) (A, B) and inactive total UC (C, D). (A, C) Staining with the neutrophil marker myeloperoxidase; $(B, D)$ staining with CD66b, used as a marker of activation. Included are two patients, representative of six patients with active total UC and five patients with inactive total UC respectively. Cells were stained with APAAP and fast red substrate and counterstained with Mayer's haematoxylin. Original magnification $\times 340$. 


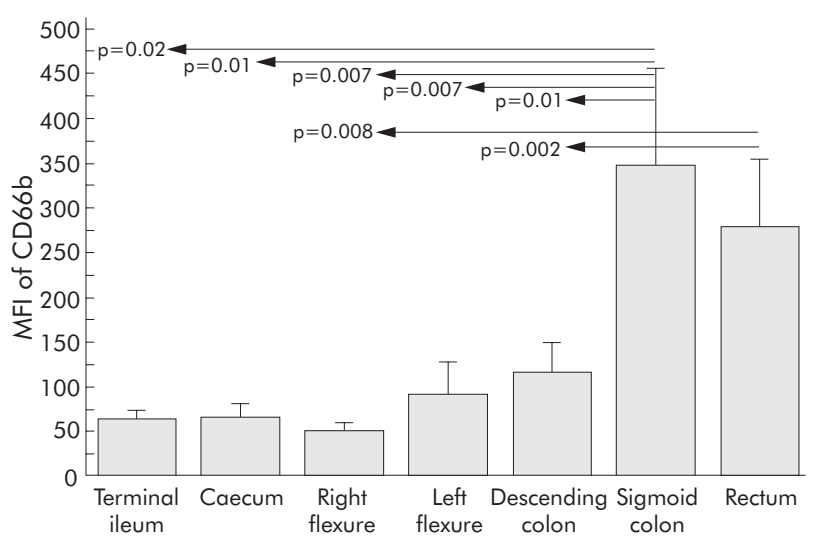

Figure 8 Mean fluorescence intensity (MFI) of CD66b on intestinal neutrophils from patients with active distal ulcerative colitis (UC) $(n=8)$. Significant differences (Friedman ANOVA and the Wilcoxon matched pairs test) between different intestinal locations are indicated. Results are expressed as mean (SEM).

the colon and terminal ileum in these patients was as low as in the control subjects (fig 8).

\section{Peripheral blood neutrophils}

No significant difference in expression of CD66b on peripheral blood neutrophils was found between control subjects and patients, either with active or inactive disease (not shown).

\section{DISCUSSION}

We have established a method for characterisation of immunological and inflammatory cells in the intestinal mucosa based on flow cytometry. The method allows us to evaluate the cell distribution and activation in biopsy specimens and is a valuable tool for assessment of immunological events in the tissue. Lymphocytes from both peripheral blood and tissue are easily identified by flow cytometry, but identification of eosinophil and neutrophil granulocytes has not, to our knowledge, been well described previously. Our use of gating and cell surface markers makes it possible to study granulocytes at the inflammatory site without permeabilising the cells. This in turn allows sorting of biopsy granulocytes for functional studies and cell culture. We found that flow cytometry is a stable and sensitive method for assessment of cell activation. It is not optimal however for comparative quantification of granulocytes between different samples. For this purpose, immunohistochemistry was used as a complementary method.

The results of this study confirm earlier findings of neutrophil involvement in active UC. ${ }^{30-32}$ Immunohistochemical staining with MPO revealed dense neutrophil infiltration in the colonic mucosa from patients with active UC but only few neutrophils in that from patients with inactive UC. We have also demonstrated that neutrophils are highly activated during the active phase of the disease whereas during inactive UC these cells express a resting phenotype. Our results from patients with distal UC show that neutrophil activation is restricted to the focus of inflammation, with increased CD66b expression only in the sigmoid colon and rectum. Taken together, these results confirm the view of the neutrophil granulocyte as an effector cell in UC that apparently plays no role during inactive disease.

The role of eosinophil granulocytes in UC, on the other hand, is not as clearcut. In normal non-inflamed intestine there is a baseline level of eosinophils that are resident in the lamina propria which may be regulated by eotaxin together with IL-5. ${ }^{33}$ During inflammation, eosinophils are highly increased in number. Morphological and immunohistochemical studies have revealed activation of intestinal eosinophils in inflammatory disease, ${ }^{3435}$ and increased intraluminal release of ECP, EPO, and EPX has been observed in active UC. ${ }^{113637}$ Our finding of increased numbers of activated eosinophils in patients with active UC compared with control subjects is in line with the view of the eosinophil as a proinflammatory cell. The decrease in CD9 expression on individual eosinophils confirms that the change in the percentage of activated cells is not due to an overall change in the number of eosinophils but is a true reflection of eosinophil activity.

The results of our comparisons of patients with active UC and control subjects might have led us to conclude that the role of eosinophils in UC is similar to that of neutrophils. However, inclusion of patients with inactive UC gave us the possibility of studying immunological conditions during quiescent disease. We found that the activity of eosinophils was higher during the inactive phase than during inflammation, both when measured by the proportion of $\mathrm{CD} 44^{+}$cells and by CD9 expression. This novel observation suggests that eosinophils may be involved in the resolution of inflammation and repair of damaged intestinal tissue. Recently, there have been reports on the role of eosinophils in tissue remodelling and repair in allergy and asthma, both in humans and in animal models. ${ }^{10} 3839$ These studies showed that eosinophil derived TGF- $\beta 1$ and IL-13 promote the transformation of fibroblasts to myofibroblasts, and stimulate expression of tenascin and procollagen I by these cells. IL-5 was found to have a critical role in this process.

Stenfeldt and Wenneras observed that eosinophils recognise and are activated by danger signals released from damaged epithelial cells. ${ }^{40}$ They found that damaged HT29 cells (an intestinal neoplastic epithelial cell line) were very potent in stimulating eosinophil chemotaxis and release of granule proteins, as well as of fibroblast growth factor 1 and TGF- $\beta$.

Thus eosinophils appear to have the capacity for both tissue destruction and repair, and may exert different actions in different stages of UC, possibly depending on what stimulus or drug they are exposed to. Another aspect of eosinophil TGF- $\beta 1$ production that has to be taken into account is that uncontrolled expression of factors stimulating fibroblast activity may lead to fibrosis, a phenomenon described in both asthma and inflammatory bowel disease. ${ }^{41}{ }^{42}$ We have also shown in this study that the activity of eosinophils, in contrast with that of neutrophils, does not appear to be restricted to sites of active inflammation. Although the percentage of activated eosinophils was significantly higher in the rectum of patients with distal colitis, we also found higher proportions of activated eosinophils in more proximal parts of the colon compared with controls. This is in agreement with previous reports of high eosinophil counts in both inflamed and non-inflamed intestinal tissue from patients with Crohn's disease, ${ }^{35}$ and in apparently healthy mucosa from resection margins of UC specimens. ${ }^{43}$ Elevated intraluminal levels of EPX have been detected in the sigmoid colon of patients with isolated proctitis, indicating eosinophil activity in the proximal colon. ${ }^{12}$ A possible interpretation of these findings is that eosinophils may have a role in the propagation of the inflammation and in the genesis of early lesions. If this is the case, the level of eosinophil activation in apparently normal mucosa could be a predictor of spreading disease. Intriguingly, there was also a high degree of eosinophil activity in the terminal ileum of patients with distal colitis. This part of the intestine is by definition not affected in UC, and the activity cannot be explained by the phenomenon 
"back wash ileitis" in patients with distal colitis. At this point, we cannot speculate on this issue but further investigation may be of interest.

In conclusion, the proinflammatory role of neutrophils in UC has been further established by this method. Flow cytometry proved to be a very useful means of studying tissue cells, and in particular cell activation. Our findings of eosinophil activity during inactive UC and in apparently unaffected parts of the colon in distal colitis generate several questions: is the eosinophil mainly involved in tissue destruction, tissue repair, fibrosis, or all of these processes? Are granulocytes present in early events of incident UC or are they recruited at a later stage? Do they participate in the pathogenesis of the disease or do they protect already injured epithelium against infectious agents? In the search for answers to these questions, we are currently performing functional studies on intestinal neutrophils and eosinophils from patients with UC in different stages of the disease.

\section{ACKNOWLEDGEMENTS}

We acknowledge the skilful technical assistance of Ingrid Stolt and the personnel at the Endoscopy Unit.

\section{Authors' affiliations}

M Lampinen, A Rönnblom, G Kristjansson, F Rorsman, P Sangfelt,

B Säfsten, M Wagner, M Carlson, Department of Medical Sciences,

Gastroenterology Research Group, University Hospital, Uppsala,

Sweden

K Amin, Department of Medical Sciences, Clinical Chemistry, University Hospital, Uppsala, Sweden

A Wanders, Department of Genetics and Pathology, University Hospital, Uppsala, Sweden

O Winqvist, Department of Medical Sciences, Immunotherapy Research Group, University Hospital, Uppsala, Sweden

Conflict of interest: None declared.

\section{REFERENCES}

1 Riddell RH. Pathology of idiopathic inflammatory bowel disease. In: Sartor RB Sandborn WJ, eds. Inflammatory bowel diseases. Edinburgh: WB Saunders, 2004:399-424.

2 Weller PF, Rand TH, Barrett T, et al. Accessory cell function of human eosinophils. HLA-DR-dependent, MHC-restricted antigen-presentation and IL1 alpha expression. J Immunol 1993; 150:2554-62.

3 Levi-Schaffer F, Barkans J, Newman TM, et al. Identification of interleukin-2 in human peripheral blood eosinophils. Immunology 1996:87:155-61.

4 Dubucquoi S, Desreumaux P, Janin A, et al. Interleukin 5 synthesis by eosinophils: association with granules and immunoglobulin-dependent secretion. J Exp Med 1994;179:703-8.

5 Nakajima H, Gleich GJ, Kita H. Constitutive production of IL-4 and IL-10 and stimulated production of IL-8 by normal peripheral blood eosinophils. $\mathrm{J}$ Immunol 1996;156:4859-66.

6 Venge P, Bergstrand H, Håkansson L. Neutrophils and eosinophils. In: Kelley WN, Harris ED, Ruddy S, et al. Textbook of rheumatology. Philadelphia: WB Saunders Co, 1996:146-60.

7 Woerly G, Roger N, Loiseau S, et al. Expression of CD28 and CD86 by human eosinophils and role in the secretion of type 1 cytokines (interleukin-2 and interferon- $\gamma$ ): inhibition by immunoglobulin A complexes. J Exp Med 1999; 190:487-95.

8 Rothenberg ME, Mishra A, Brandt EB, et al. Gastrointestinal eosinophils. Immunol Rev 2001;179:139-55.

9 Zagai U, Skold CM, Trulson A, et al. The effect of eosinophils on collagen gel contraction and implications for tissue remodelling. Clin Exp Immunol 2004; 135:427-33.

10 Phipps S, Ying S, Wangoo A, et al. The relationship between allergen-induced tissue eosinophilia and markers of repair and remodeling in human atopic skin. J Immunol 2002;169:4604-12.

11 Bischoff SC, Wedemeyer J, Herrmann A, et al. Quantitative assessment of intestinal eosinophils and mast cells in inflammatory bowel disease. Histopathology 1996;28:1-13.

12 Carlson M, Raab Y, Peterson C, et al. Increased intraluminal release of eosinophil granule proteins EPO, ECP, EPX, and cytokines in ulcerative colitis and proctitis in segmental perfusion. Am J Gastroenterol 1999;94:1876-83.

13 Peterson CGB, Eklund E, Taha $Y$, et al. A new method for the quantification of neutrophil and eosinophil cationic proteins in feces: establishment of normal levels and clinical application in patients with inflammatory bowel disease. Am J Gastroenterol 2002;97:1755-62.

14 Lampinen M, Carlson M, Sangfelt P, et al. IL-5 and TNF- $\alpha$ participate in recruitment of eosinophils to intestinal mucosa in ulcerative colitis. Dig Dis Sci 2001:46:2004-9.

15 Koizumi M, King N, Lobb R, et al. Expession of vascular adhesion molecules in inflammatory bowel disease. Gastroenterology 1992;103:840-7.

16 Elliott SN, Wallace JL. Neutrophil-mediated gastrointestinal injury. Can J Gastroenterol 1998;12:559-68.

17 Panés J, Granger DN. Leukocyte-endothelial cell interactions: molecular mechanisms and implications in gastrointestinal disease. Gastroenterology 1998; 114:1066-90.

18 Carlson M, Raab Y, Sevéus L, et al. Human neutrophil lipocalin is a unique marker of neutrophil inflammation in ulcerative colitis and proctitis. Gut 2002;50:501-6.

19 Izzo RS, Witkon K, Chen Al, et al. Interleukin-8 and neutrophil markers in colonic mucosa from patients with ulcerative colitis. Am J Gastroenterol 1992;87: 1447-52.

20 Sands BE. From symptom to diagnosis: clinical distinctions among various forms of intestinal inflammation. Gastroenterology 2004;126:1518-32.

21 Binder V. A comparison between clinical state, macroscopic and microscopic appearances of rectal mucosa, and cytologic picture of mucosal exudate in ulcerative colitis. Scand J Gastroenterol 1970;5:627-32.

22 Fernvik E, Halldén G, Hed J, et al. Intracellular and surface distribution of CD9 in human eosinophils. APMIS 1995;103:699-706.

23 Upham JW, Sehmi R, Hayes LM, et al. Retinoic acid modulates IL-5 receptor expression and selectively inhibits eosinophil-basophil differentiation of hemopoietic progenitor cells. J Allergy Clin Immunol 2002;109:307-13.

24 Matsumoto K, Appiah-Pippim J, Schleimer RP, et al. CD44 and CD69 represent different types of cell-surface activation markers for human eosinophils. Am J Respir Cell Mol Biol 1998;18:860-6.

25 Fernvik E, Lundahl J, Halldén G. The impact of eotaxin- and IL-5-induced adhesion and transmigration on eosinophil activity markers. Inflammation 2000;24:73-87.

26 Bochner BS, Sterbinsky SA, Bickel CA, et al. Differences between human eosinophils and neutrophils in the function and expression of sialic acidcontaining counterligands for E-selectin. J Immunol 1994;152:774-82.

27 Stocks SC, Kerr MA, Haslett C, et al. CD66-dependent neutrophil activation: a possible mechanism for vascular selectin-mediated regulation of neutrophil adhesion. J Leuk Biol 1995;58:40-8.

28 Torsteinsdóttir I, Arvidson N-G, Hällgren R, et al. Enhanced expression of integrins and CD66b on peripheral blood neutrophils and eosinophils in patients with rheumatoid arthritis, and the effect of glucocorticoids. Scand J Immunol 1999;50:433-9.

29 Amin K, Rinne J, Haahtela T, et al. Inflammatory cell and epithelial characteristics of perennial allergic and nonallergic rhinitis with a symptom history of 1 to 3 years' duration. J Allergy Clin Immunol 2004;107:249-57.

30 Madara JL. Review article: Pathobiology of neutrophil interactions with intestinal epithelia. Aliment Pharmacol Ther 1997;11:57-63

31 Raab Y, Gerdin B, Ahlstedt S, et al. Neutrophil mucosal involvement is accompanied by enhanced local production of interleukin- 8 in ulcerative colitis. Gut 1993;34:1203-6.

32 Robinson CE, Kottapalli V, D'Astice M, et al. Regulation of neutrophils in ulcerative colitis by colonic factors: a possible mechanism of neutrophil activation and tissue damage. J Lab Clin Med 1997;130:590-602.

33 Mishra A, Hogan SP, Lee JJ, et al. Fundamental signals that regulate eosinophil homing to the gastrointestinal tract. J Clin Invest 1999; 103:1719-27.

34 Dubucquoi S, Janin A, Klein O, et al. Activated eosinophils and interleukin-5 expression in early recurrence of Crohn's disease. Gut 1995;67:242-6.

35 Carvalho AT, Elia CC, De Souza HS, et al. Immunohistochemical study of intestinal eosinophils in inflammatory bowel disease. J Clin Gastroenterol 2003;36:120-5.

36 Makiyama K, Kanzaki S, Yamasaki K, et al. Activation of eosinophils in the pathophysiology of ulcerative colitis. J Gastroenterol 1995;30:64-9.

37 Sangfelt $\mathbf{P}$, Carlson $M$, Thörn $M$, et al. Neutrophil and eosinophil granule proteins as markers of response to local prednisolone treatment in distal ulcerative colitis and proctitis. Am J Gastroenterol 2001;96:1085-90.

38 Flood-Page P, Menzies-Gow A, Phipps RP, et al. Anti-IL-5 treatment reduces deposition of ECM proteins in the bronchial subepithelial basement membrane of mild atopic asthmatics. J Clin Invest 2003;1 12:1029-36.

39 Cho JY, Miller M, Baek KJ, et al. Inhibition of airway remodeling in IL-5deficient mice. J Clin Invest 2004; 1 13:551-60.

40 Stenfeldt AL, Wenneras C. Danger signals derived from stressed and necrotic epithelial cells activate human eosinophils. Immunology 2004;112:605-14.

41 Levi-Schaffer F, Garbuzenko E, Rubin A, et al. Human eosinophils regulate human lung- and skin-derived fibroblast properties in vitro: a role for transforming growth factor beta (TGF- $\beta$ ). Proc Natl Acad Sci U S A 1999;96:9660-5

42 Ståhle-Bäckdahl M, Malm J, Veress B, et al. Increased presence of eosinophilic granulocytes expressing transforming growth factor- $\beta 1$ in collagenous colitis. Scand J Gastroent 2000;35:742-6.

43 Jeziorska M, Haboubi N, Schofield P, et al. Distribution and activation of eosinophils in inflammatory bowel disease using an improved immunohistochemical technique. J Pathol 2001;194:484-92. 